\title{
L'AGE D'OR DES BACHAREIS? LES ELITES POLITIQUES FEDERALES DE LA PREMIERE REPUBLIQUE BRESIL, 1889-1930
}

\author{
Armelle Enders
}

Université de Paris IV

\section{RÉSUMÉE}

Les études portant la Première République brésiliène $(1889$ - 1930), se sont concentrées sur le coronelismo, comme une forme de privatisation de l'appareil d'État, mais ils ont négligé d'autres lieux et figures du pouvoir comme le bacharelismo, le quel, selon certains historiens, était une des caracteristiques plus importantes du Brésil de l'époque. Les bacharéis (bacheliers en droit) faisaient part d'une minorité de Brésiliens ayant réçu une formation supérieure et occupant une place préponderante dans le affaires de l'État. Le bacharelismo finit aussi par caractériser toute une culture, laquel se traduit par la réthorique, les hummanités classiques et la méconnaissance des réalités nationales. 
L'árticle fait un étude du parcours des bacharéis de l'Empire jusqu'a la Première République, à fin d'évaluer le poids du bacharelismo dans la culture Brésilienne.

\section{RESUMEN}

El estudio de la Primera República brasileña (1889 - 1930) se ha centrado principalmente en el coronelismo, como una forma de privatización del aparato estatal a favor de los gamonales locales y de sus clientelas. Sin embargo, se han descuidado otros fenómenos como el bacharelismo, que de acuerdo a algunos autores constituía una de las características más importantes del Brasil de la época. Los bacharéis (bachilleres graduados en derecho) pertenecían a una minoría de brasileños que recibieron una formación superior y ocupaban altos puestos en el Estado. El bacharelismo llegó también a significar toda una cultura caracterizada por el uso de la retórica, las humanidades clásicas y la falta de conocimientos sobre la realidad nacional.

El artículo analiza el recorrido de los bacharéis, desde los últimos años del Imperio hasta el final de la Primera República, con el fin de evaluar su peso dentro de la cultura brasileña.

Les études portant la vie politique brésilienne se sont plus volontiers concentrées sur la figure du coronel, le maitre des campagnes, que sur celle du bachárel, le diplômé des villes. À la suite de l'ouvrage classique de Vítor Nunes Leal, Coronelismo, enxada e votos, publié en 1949, la Première République est lue en général comme le berceau d'un système dont les prolongements se font encore sentir dans le Brésil d'aujourd' hui. Selon cet auteur, avec la constitution républicaine de 1891, des propriétaires fonciers et des commerçants frappés par le déclin économique ont pu, grâce au contrôle des électeurs et des élections, sauver une situation économique compromise et consolider leur domination locale. Le nom générique de coronel (pl. coronéis) renvoie aux grades d'officiers de la Garde Nationale, peu à peu vidées de contenu, mais dont le prestige demeurait intact auprès des petits notables de l'intérieur.
Le coronelismo, forme de privatisation de l'appareil d'État au profit des coqs de village et de leurs clans, a été ainsi érigé en concept, âprement débattu depuis lors.' Quant au coronel "de l'intérieur", il a été promu en type social éternel.

La masse d'informations et de réflexions consacrées au coronelismo rural éclipse pourtant d'autres lieux et figures du pouvoir sous la Première République. Les formes urbaines du coronelismo, décelable par exemple dans les soubresauts du Distrito Federal (Rio de Janeiro-ville), ${ }^{2}$ ont été négligées, comme l'ont été les élites fédérales, distinctes à bien des égards des coronéis provinciaux, et pourvoyeuses, autant que ces derniers, de modèles politiques, sociaux et culturels. Une dizaine d'années avant Vítor Nunes Leal, dans Raizes do Brasil (1936), Sérgio Buarque de Holanda faisait du bacharelismo une des caractéristiques du Brésil contemporain. Dans ce texte, l'historien brésilien thématisait une expression triviale et plutôt péjorative. Après la Révolution de 1930, la Vieille République et le bacharelismo étaient en effet confondus dans un même rejet. Le terme de bacharel désigne au sens strict le bachelier en droit et, par extension, la minorité de Brésiliens ayant reçu une formation supérieure et occupant une place prépondérante dans les affaires de l'État.

Le bacharelismo finit par résumer une tournure d'esprit particulière de certaines élites brésiliennes, laquelle se traduirait par un penchant pour la rhétorique, les humanités classiques, les constructions théoriques et la méconnaissance des réalités nationales. Il faut distinguer les bacharéis, groupe social, du bacharelismo, une culture spécifique marquée, selon Sérgio Buarque de Holanda, "par un certain

\footnotetext{
VItor Nunes Leal, Coronelismo, enxada e votos: o mumicipio e o regime representativo no Brasil, São Paulo, 1949. Le débat a été poursuivi par Raimundo Faoro, Os donos do poder. São Paulo. 1958: Maria Isaura Pereira de Queiroz. "O coronelismo numa interpretação sociológica", S. Buarque de Holanda \& B Fausto, eds., História Geral da Civilização Brasileira, vol. 3, t. 1, 1976, pp. 153-187; Paul Cammack, "O 'coronelismo' e o 'compromisso coronelista': uma critica", Cadernos DCP. Belo Horizonte, No. 5 1979, pp. 5-20; Eul Soo Pang, Coronelismo e oligarquias 1889-1934. Rio de Janeiro, 1979; José Murito de Carvalho, "Coronelismo", A. Abreu \& I. Beloch, eds., Dicionário histórico-biográfico brasilerro 1930-1983. Rio de Janeiro, Forense, 1983; Amilcar Viana Martins Filho, "Clientelismo e representaçào emi Minas Gerais durante a Primeira República: uma critica a Paul Cammack", Dados, Rio de Janeiro, vol. 27, No. 2, 1984, pp. 175-197; José Murilo de Carvalho, "Mandonismo, clientelismo, coronelismo: uma discussa conceitual", Pontos e bordados. Escritos de história e politica, Belo Horizonte, ed. UFMG, 1998, pp. 130-153.

Américo Oscar Guichard Freire, Uma capital para a Repuiblica. Poder federal e forças locais no campo político carioca (1889-1906), tese de doutoramento, IFCS-Universidade Federal do Rio de Janeiro, 1998
} 
type d'érudition formelle et de façade, où les mots rares, les épithètes supposées scientifiques, les citations en langue étrangère, sont destinés à éblouir le lecteur, comme le ferait une collection de pierre précieuses rutilantes." ${ }^{n}$ Pour utiliser une facilité offerte par la traduction, un bachelier en droit n'a pas nécessairement un comportement de bacharel, de même que le bacharelismo n'est pas le seul fait des diplômés en droit.

Le parcours des bacharéis sous la Vieille République fournit l'occasion de comparer le personnel politique républicain et celui de l'Empire, et d'évaluer le poids du bacharelismo dans la culture brésilienne.

\section{LA FABRIQUE DES BACHAREIS}

Une des spécificités de la domination coloniale portugaise consiste à avoir interdit les établissements d'enseignement supérieur dans ses possessions d'outre-mer. Le service de la Couronne était largement ouvert aux sujets nés au Brésil ou dans d'autres parties de l'empire, pour peu qu'ils aient été formés auparavant à l'université de Coimbra. Les études au Portugal garantissaient une situation privilégiée dans la société coloniale et métropolitaine. Envoyer ses enfants étudier à Coimbra justifiait donc bien des sacrifices de la part des grandes familles de l'Amérique portugaise. Les étudiants originaires de l'Estado do Brasil formaient ainsi au Portugal des contingents importants, tant par leur nombre que par les distinctions qu'ils y glânaient. ${ }^{4}$ Les "Lusobrésiliens" diplômés à Coimbra jouent un rôle crucial dans l'administration portugaise, le transfert de la monarchie à Rio de Janeiro en 1808 et la formation de l'État au Brésil sous les règnes de dom João VI et de dom Pedro I. L'Université de Coimbra avait servi de creuset où s'amalgamaient les élites du monde portugais dans une même fidélité à la Couronne. Elle fut aussi à l'origine de la formation des cadres du nouvel Empire du Brésil.

Sérgio Buarque de Holanda, Racines du Brésil, Paris, Gallimard. 1998, pp. 262 (1ère éd. brésilienne: 1936). Francisco Morais, "Estudantes brasileiros na Universidade de Coimbra (1772-1872)", Anais da Biblioteca Nacional, Rio de Janeiro, vol. LXII, 1940, Rio de Janeiro, Imprensa Nacional.
Par nécessité, le régent dom João crée, dès les premières années de son séjour au Brésil, deux facultés de médecine, l'une à Salvador da Bahia et l'autre à Rio de Janeiro, ainsi qu'une académie militaire et une académie de Marine, mais il faut attendre l'indépendance du Brésil pour voir apparaître, à São Paulo et à Recife (puis Olinda), deux facultés de droit en $1827 .^{5}$ En ce qui concerne cette discipline, les Brésiliens ne perdent vraiment le chemin de Coimbra qu'après 1850.

José Murilo de Carvalho, auteur d'une thèse sur le personnel politique et administratif de l'Empire, souligne l'importance de ce modèle de Coimbra et des facultés de droit dans l'homogénéité de l'élite politique impériale.6 Sous l'Empire en effet, les trois quart des ministres ont reçu une formation juridique et sont, à l'origine, fonctionnaires, magistrats ou avocats. La bureaucratie impériale, composée de lettrés dans un pays où le taux d'analphabétisme dépasse à l'époque les $80 \%$, constitue un groupe très spécifique. La carrière dans la fonction publique attire, selon José Murilo de Carvalho, les "marginaux du système agraroesclavagiste", ceux qui n'y ont pas trouvé leur place ou qui l'ont perdue, les senhores de engenho en crise, qui cherchent dans la fonction publique une nouvelle rente. Les propriétaires fonciers sont une infime minorité à faire partie des élites politiques impériales. Selon l'historien, $95 \%$ des ministres de Dom Pedro II, 90\% des députés, $85 \%$ des sénateurs, $100 \%$ des conseillers d'Etat, sont issus des quelques 16.000 Brésiliens qui sont magistrats, avocats, médecins, professeurs, prêtres, ou hommes de lettres. Les militaires et les fazendeiros sont en revanche peu nombreux. ${ }^{7}$ Au cours du XIX ${ }^{\mathrm{e}}$ siècle, les promotions de juristes ne cessent de s'étoffer. Pendant la décennie 1830 , un peu plus de 700 lauréats sortent des facultés de droit du Brésil. Entre 1880 et 1889, ils sont presque 2.000 .

Cette croissance numérique, plus rapide que celle des débouchés qui étaient offerts à ces diplômés, aurait contribué à les détacher de la monarchie et à faciliter l'instauration de la République. ${ }^{8}$ Le décret signé en 1891 par Benjamin Constant

Voir Alberto Venàncio Filho, Das arcadas ao bacharelismo Så Paulo Perspectiva "Estudos", 1982 Voir José Murilo de Carvalho, A construção da ordem: a elite politica imperial, Rio de Janeiro, Campus, 1980. José Murilo de Carvalho, $A$ construção da ordem: a elite politica imperial, Rio de Janeiro, Campus, 1980, p. 74 Richard Graham, Patronage and Politics in Nineteenth-Centriry Brazil. Stanford, Stanford University Press, 1990, p. 266. 
Botelho de Magalhães, ministre du gouvernement provisoire, autorise la fondation de facultés libres dans les États de la nouvelle République fédérative. Les cours de droit se multiplient dans les capitales du Brésil. Les diplômés aussi.

\section{JURIDISME ET VERBALISME}

Le passage par les facultés vaut moins par l'enseignement qui y est prodigué que par la socialisation qu'elles offrent à ses élèves. La correspondance diplomatique française offre un témoignage sur la qualité de la formation qu'on reçoit dans ces écoles où les diplômes, "libéralement prodigués à des élèves dispensés le plus souvent de suivre les cours par des professeurs dont la "bonhommie" est légendaire, ne sauraient être la garantie d'une instruction solide ou d'une aptitude professionnelleéprouvée. ${ }^{\prime 9}$ Certains n'hésitent pas d'ailleurs à accumuler les cursus et les parchemins. Henrique Dodsworth (1895-1975), député du District fédéral, et futur prefeito de Rio sous Vargas, est un ancien élève des facultés de médecine et de droit de Rio de Janeiro; Gilberto Amado (1887-1969), député du Sergipe, enchaine des études médicales et des études de droit, tout cela pour se consacrer un peu à l'enseignement, beaucoup au journalisme et à la politique. ${ }^{10}$ Quant à Júlio Brandão, le seul homme politique mineiro important qui n'ait pas reçu cette forme d'adoubement universitaire, il se forma en droit et exerça comme avocat dans le Minas Gerais, ce qui prouve que, pour des fins strictement professionnelles, on pouvait se dispenser de l'enseignement des facultés. ${ }^{.1}$

Un diplôme est plus un titre nobiliaire que la garantie d'une compétence. Pour les générations formées sous l'Empire et qui disparaissent de la scène politique dans les années 1920, les facultés de droit de São Paulo et de Recife sont un passage quasi obligé de l'initiation d'un jeune homme bien né ou promis à une belle carrière.

Ministère des Affaires Etrangères, Correspondance Politique, Brésil, 30 juillet 1892. f. 203

to Alzira de Abreu et Israel Beloch, Dicionário histórico-biográfico brasileiro 1930-1983, Rio de Janeiro Forense, 1983 .

Amilcar Viana Martins Filho, The White Collar Republic: Patronage and Interest Representation in Minas Gerais, 1889-1930, Ph.D. University of Illinois, 1987, p.113. Sur la règlementation des professions libérales, voir Edmundo Campos Coelho, As profissões imperiais. Medecina, Engenharia e Advocacia no Rio de Janeiro, 1822-1930, Rio de Janeiro, Record, 1999.
Dans sa biographie du président Rodrigues Alves, ${ }^{12}$ l'écrivain et homme politique Afonso Arinos de Melo Franco s'attarde longuement sur la vie estudiantine à São Paulo autour de 1870. On entre vers 14-15 ans à la Faculté où l'on reste 3 ou 4 ans. La promotion de Rodrigues Alves à la Faculté de Droit de São Paulo est une ébauche de panthéon national: Rui Barbosa, Afonso Pena, Joaquim Nabuco, et Castro Alves en font partie. La littérature et la politique tiennent une grande place dans les activités des apprentis juristes de São Paulo. Avant 1870, jeunes libéraux et jeunes conservateurs s'empoignent dans de bouillants articles, confrontent leurs talents oratoires, entrent dans des loges maçonniques ou dans d'autres sociétés secrètes. Une des associations secrètes les plus durables et les plus importantes est la Burschenschaft, qui, d'après Afonso Arinos de Melo Franco, avait été fondée vers 1834 par un exilé politique allemand et s'était perpétuée avec succès..$^{13}$ Par son fondateur et son histoire supposée, la Burschenschaft se rattachait aux luttes libérales et nationalistes pour l'unité de l'Allemagne du début du XIXe siècle. L'organisation cooptait ses membres et obéissait à une hiérarchie précise. Ceux qui en furent les chefș lors de leur passage à la Faculté de São Paulo jouent les premiers rôles sous la République: les futurs présidents Rodrigues Alves, Afonso Pena, Campos Sales, Venceslau Brás, Washington Luís, Artur Bernardes, le futur ministre des affaires étrangères baron de Rio Branco, les futurs ministres et chefs politiques Rui Barbosa, Pinheiro Machado, Bernardino de Campos, Davi Campista.

Sans voir dans la Burschenschaft, comme le suggère Afonso Arinos, une armature occulte qui aurait prolongé dans la République son processus de cooptation et aurait contribué ainsi à maintenir la cohérence des élites politiques, on peut l'interpréter comme un résumé particulièrement condensé de la société politique brésilienne. La Burschenschaff est en quelque sorte une redondance de la Faculté de São Paulo, une cooptation à l'intérieur de la cooptation. Elle constitue un avant goût de franc-maçonnerie, tradition prospère des élites brésiliennes depuis la fin du XVIII siècle. Les rituels folkloriques et la camaraderie de monôme qui en résulte ne font que symboliser l'étroitesse du milieu d'où sortent les élites politiques, l'appartenance à un même monde et la même vocation à exercer un jour le pouvoir.

12 Afonso Arinos de Melo Franco, Rodrigues Alves, Rio de Janeiro, ed. USP, 1973.

13 Sur la Burschenschaft, voir Afonso Arinos de Melo, Rodrigutes Alves. Rodrigues Alves, Rio de Janeiro, ed. USP, 1973, pp. $24-35$. 
Les générations suivantes fréquentent plus volontiers les facultés créées dans leurs Etats respectifs, mais reproduisent à l'échelle régionale les traditions de cooptation et de sociabilité des vieilles facultés fédérales. Les nouveaux établissements, pépinières d'assesseurs et de successeurs, sont d'ailleurs patronnés par les dirigeants politiques locaux qui y enseignent souvent. Les échanges de discours et d'hommages entre les élus et la "mocidade acadêmica" la jeunesse des écoles, sont monnaie courante. José Joaquim Seabra, adversaire politique de Rui Barbosa à Bahia, tente de rivaliser avec ce dernier dans le cœur des étudiants bahianais. Il choye l'école de médecine qu'il fait reconstruire après un incendie, ce qui lui vaut d'avoir son buste en bronze exposé dans le salon d'apparat.14

Entre l'Empire et la République, la différence la plus frappante semble plutôt la multiplication et la dispersion des facultés qu'un véritable changement dans la formation des élites politiques. L'effet burschenschaft tend à se diluer dans le fédéralisme au fur et à mesure que celui-ci s'implante au Brésil.

\section{"BUREAUCRATIE IMPERIALE", ELITES FEDERALES REPUBLICAINES, LA CONTINUITE PAR LES BACHAREIS}

La comparaison entre les serviteurs de l'État impérial et le personnel politique de la Première République appartient aux topoi à la fois des polémiques des années 1920 , période où le régime fait l'objet de sévères évaluations, et des controverses universitaires contemporaines. En 1924 par exemple, deux contributions issues d'un même ouvrage portent un diagnostic diamétralement opposé sur les élites au pouvoir. Francisco de Oliveira Viana, essayiste réactionnaire, voit un vice originel dans le fait d'avoir substitué aux élites impériales, c'est-à-dire "ce qu'il y avait de plus prestigieux et de fin dans la vieille aristocratie locale", de nombreuses "entités interlopes, très arrivistes, très nouveaux riches politiques, au caractère peu solide et à la conscience peu scrupuleuse."15 C'est un tout autre avis qu'émet, dans le

14 Voir Francisco Borges de Barros, Dr. José Joaquim Seabra: sta vida, sta obra na Repriblica. Bahia, Imprensa Oficial do Estado, 1931, p. 494.

15 En français dans le texte. Francisco Oliveira Viana, "O idealismo da Constituição", Vicente Licínio, ed., A margem da Repriblica, Brasilia, ed. Universidade federal de Brasilia, 1981, (1 ère éd.: 1924), p. 104. même ouvrage collectif, l'homme politique et écrivain Gilberto Amado (18871969), selon lequel "la République ne saurait être autre chose que, comme l'était la monarchie, les avocats qui sortent des Facultés de Droit chaque année par centaines; les médecins, les ingénieurs, les docteurs, les anciens seigneurs, enfin, en la personne de leurs descendants ruinés; les fils des esclaves, des métis, des mulâtres placés dans la fonction publique et dans les carrières libérales."96

Pour le premier auteur, le changement d'élite traduit la décadence politique du Brésil. Le second estime en revanche que la permanence au pouvoir d'élites produites par la société impériale creuse l'écart entre le pays légal et le pays réel et ne correspond pas à la base sociale du régime républicain. La citation de Gilberto Amado présente en outre l'intérêt de résumer la complexité et l'ambiguïté du monde politique et administratif brésilien, tant de la période impériale que républicaine. On y perçoit parfaitement l'importance des diplômes le rôle du clientélisme et du patronage, des élites sociales relativement variées.

Hormis quelques mises à l'écart, quelques promotions subites et quelques retraites boudeuses, la Proclamation du 15 novembre 1889 n'a pas entrainé de grande épuration dans les élites politiques. L'aristocratie impériale se porte bien sous la République, dans les anciennes "vieilles provinces" de Bahia, Rio de Janeiro et du Pernambouc. Nombre de ses rejetons appartiennent à la représentation nationale républicaine. La liste des élus bahianais de la première législature fédérale (18911893) ressemble à un résumé du gotha. On peut y lire les noms d'Antônio Joaquim Pires de Carvalho e Albuquerque, baron de Vila Viçosa, ainsi que de son fils Garcia, vicomte da Torre de Garcia de Avila, de Francisco dos Santos Pereira, fils du baron de Alagoinhas, de Joaquim Cardoso Pereira de Melo, baron de São Marcos. ${ }^{17}$ Quelques années plus tard, José Wanderley de Araújo Pinho (18901967), bacharel en droit, prefeito de Salvador, petit-fils du côté maternel du baron de Cotegipe, rejeton de quatre générations de senhores de engenho, représente Bahia à la Chambre fédérale de 1924 à 1930.

Gilberto Amado, "As instituições politicas e o meio social no Brasil", Vicente Licínio, ed., A margem da Repuiblica, Brasilia, ed. Universidade federal de Brasilia, 1981, (lère éd., 1924), p. 56.

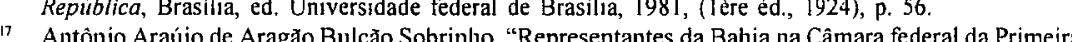
República", Revista do Institino Histórico e Geográfico da Bahia, No. 263, 1964, pp. 55-86. 
Les travaux des historiens contemporains ne s'écartent guère du schéma tracé par Gilberto Amado et Oliveira Viana et aboutissent à des conclusions contrastées. Dans une perspective weberienne, José Murilo de Carvalho défend l'idée que l'Empire a su rompre avec la patrimonialisation de l'État, en constituant, à partir d'un groupe social aux origines variées, une bureaucratie moderne, attachée à la distinction entre sphère privée et domaine public. ${ }^{18}$ Par "bureaucratie impériale", José Murilo de Carvalho entend la fonction publique -l'administration impériale compte en 1877 environ 80.000 fonctionnaires pour une population estimée à quelques 14 millions d'habitants- et le personnel politique: ministres, députés, sénateurs, membres du prestigieux Conseil d'Etat. La "bureaucratie impériale" défend et illustre les principes d'un consensus de base: le maintien de la centralisation, la méfiance à l'égard de l'armée, des prétentions sociales et politiques de celle-ci, la défense de la monarchie constitutionnelle. La composition de l'élite impériale évolue cependant quelque peu dans le dernier quart du XIX ${ }^{\mathrm{e}}$ siècle et fait une plus large place aux professions libérales. Peu représentative de la société brésilienne, la "bureaucratie" impériale est le bras administratif du pouvoir modérateur de l'empereur, défini par la constitution de 1824. Le tableau que dresse José Murilo de Carvalho peut se réduire à quelques lignes directrices. Les "bureaucrates" de dom Pedro II sont des bacharéis; ils ne sont pas directement issus des intérêts agraires; ils servent l'État et non les "petites patries" dont ils sont originaires. La fabrication de cette "noblesse de robe" est à mettre à l'actif de la monarchie, alors que la République ne serait pas parvenue au même résultat. Richard Graham, auteur d'une étude sur le clientèlisme sous l'Empire, ${ }^{19}$ nuance fortement ce modèle, en montrant que la construction de l'État moderne au Brésil s'accommode, voire se nourrit, du système de patronage et de clientèle.

Ces travaux n'ont guère d'équivalent pour la période républicaine. Personne ne s'est chargé d'une vision d'ensemble sur les ministres, les députés, les sénateurs, les présidents des États, ${ }^{20}$ œuvrant au niveau fédéral. Fédéralisme oblige, la Première

18 José Murilo de Carvalho, A consinıı̧ão da ordem: a elite politica imperial, Rio de Janeiro, Campus, 1980 19 Richard Graliam, Patronage and Politics in Nineteenth-Century Brazil, Stanford, Stanford University Press, 1990.

20 Pendant la Première République, à la tête de la plupart des États de la Fédération, se trouve un présiden èlu au suffrage masculin direct. L'usage posterieur et le désir d'éviter de possibles confusions conduit souvent à les désigner comme "gouverneurs".
République a fait l'objet de nombreuses monographies régionales dans lesquelles entre cependant l'étude du personnel politique. Le croisement de ces données compense dans une certaine mesure l'absence de synthèse et autorise quelques conclusions.

La Première République, comme l'était l'Empire, est bien un régime de diplômés. Entre 1889 et 1937, 7 hommes politiques du Pernambouc sur 10 sont passés par la Faculté de droit de Recife. ${ }^{21}$ Au Minas Gerais, 98,4\% du personnel politique sont passés par l'enseignement supérieur.22 C'est aussi le cas pour $95,6 \%$ des 115 représentants de Bahia au Congrès fédéral dont la formation est connue. ${ }^{23}$ Les résultats sont identiques en ce qui concerne les représentants au Congrès fédéral de l'État de Rio de Janeiro. Sous la République comme sous l'Empire, les élites politiques nationales constituent un îlot de lettrés régnant sur un océan d'analphabètes (entre 70 et $80 \%$ de la population), pour reprendre l'expression que José Murilo de Carvalho emploie à propos des élites impériales.

Le droit reste de loin la discipline la plus prisée des élites politiques. Parmi les élites politiques paulistas qu'il a étudiées, Joseph Love a compté $69 \%$ d'avocats. ${ }^{24}$ Plus des $2 / 3$ des députés et sénateurs fédéraux bahianais ${ }^{25}$ qui ont reçu une formation supérieure ont fréquenté des facultés de droit, $18 \%$ d'entre eux ont fait médecine, $7 \%$ sont passés par les écoles d'ingénieur, $5 \%$ sont officiers. Sur les 5 congressistes bahianais qui ne sont pas passés par ces écoles, on compte trois fazendeiros, un commerçant et un prêtre. ${ }^{26}$

21 Robert Levine, A velha i̇sina: Pernambuco na Federação brasileira, 1889-1937, Rio de Janeiro, Paz e terra, 1980 , pp. 165 et sq.

Amilcar Viana Martins Fitho, The white collar Republic: Patronage and interest representation in Minas Gerais, 1889-1930, Ph. D. University of Illinois, 1987, p. 113.

23 Les données concernant les députés et sénateurs représentant Bahia, Rio de Janeiro et le District fédéral ont été rassemblées à partir des dictionnaires biographiques et de l'Almanaque Laemmert, un anıuaire des professions. Cf. Armelle Enders, Pourvoirs et fédéralisme au Brésil (1889-1930). Université de Paris IV, Sorbonne, 1993.

2t Joseph Love, São Paulo in the Brazilian Federation. 1889-1937, Stan ford, Stanford University Press, 1980, p. 157.

25 Pour les 110 députés et sénateurs de Bahia pour lesquels on ait des données.

36 Armelle Enders, Potroirs et fédéralisme aul Brésil (1889-1930). Université de Paris IV, Sorbonne, 1993, p. 233. 
Dans l'Etat de Rio de Janeiro, la composition de l'élite politique est tout à fait comparable avec celle de Bahia: les diplômés en droit constitue plus de la moitié de la population, les médecins un peu moins d'un tiers, les ingénieurs $(9 \%)$ sont un peu plus nombreux que les militaires $(6 \%){ }^{27}$ Les représentants du District fédéral ont un profil un peu différent: un tiers de médecins, un peu moins d'un tiers de juristes, $1 / 5$ de militaires, $1 / 10$ d'ingénieurs. ${ }^{28}$

Ces variations régionales sont délicates à interpréter. La capitale fédérale concentre sans doute un plus grand choix d'écoles renommées, en particulier l'Ecole de médecine, l'Ecole polytechnique et l'Ecole militaire qui continuent à attirer la jeunesse du pays tout entier. Le droit n'est donc pas la seule voie d'excellence proposée à la bourgeoisie carioca d'origine ou d'adoption. De plus, médecins et ingénieurs ont été à l'honneur dans les transformations successives qui font de la Capitale fédérale une ville moderne dans la première moitié du XX ${ }^{e}$ siècle. Rio de Janeiro est, depuis 1880 , le siège du Clube de engenharia dont l'objectif est de rassembler les ingénieurs et les industriels. De 1903 à 1933, c'est le sénateur carioca André Paulo de Frontin, -un des "patrons politiques" de la ville-, qui le préside. Frontin a participé à la réforme urbaine de Francisco Pereira Passos sous la présidence de Rodrigues Alves (1902-1906). ${ }^{29}$

La médecine offre d'autre part un moyen commode de se forger une clientèle, à tous les sens du terme, et d'accéder à la vie politique. L'exemple le plus achevé d'une telle carrière à Rio de Janeiro est celui de Pedro Ernesto Batista (18841942), élu (indirectement) maire de la capitale en 1934. Excellent praticien, Pedro Ernesto s'était attiré non seulement de nombreuses sympathies parmi la bonne société carioca, mais aussi une immense popularité dans lesfavelas dont il soignait gratuitement les habitants..$^{30}$ L'ascension du "bon docteur" dans la vie politique carioca s'inscrit toutefois dans une double transition. Pedro Ernesto a en effet

27 Sur les 97 députés ou sénateurs fédéraux fluminenses qui ait reçu une formation supérieure et pour lesquels on ait des données, ibidem.

28 Pour les 70 (sur les 75 recensés) pour lesquels on ait des données, ibidem.

29 Francisco Pereira Passos (1836-1913), l'ingénieur et le préfet de Rio le plus célèbre de la Première Republique, était le fils du baron de Mangaritiba, caféiculteur de Rio de Janeiro.

so Michael L. Conniff, Urban politics in Brazil. The rise of populism, 1925-1945, Pittsburgh, University of Pittshurgh Press, 1981; et Carlos Eduardo B. Sarmento, Autonomia e participação. O Partido Autonomista do Distrito Federal e o campo politico carioca (1933-1937), dissertação de mestrado, Universidade Federal do Rio de Janeiro-IFCS, 1996. pour mission de consolider dans la capitale fédérale la victoire du gouvernement issu de la Révolution de 1930 sur les décombres de la Première République. À ce changement conjoncturel s'ajoute la participation croissante des masses populaires à la vie publique. Le "bon docteur" atteint les sommets au moment même où la figure du bachárel ne correspond plus ni à la sociologie d'un électorat élargi, ni aux aspirations du Brésil des années 1930.

Des nuances différentes colorent donc le profil du personnel politique envoyé par chaque État de la Fédération dans la capitale pour le représenter et veiller aux destinées de l'Union. Les médecins et les ingénieurs sont plus nombreux à s'assoir sur les bancs réservés à la représentation du District fédéral que la moyenne des autres États, et, les gaúchos, fidèles à la tradition de province-frontière du Rio Grande do Sul, élisent un contingent non négligeable de militaires. Ces variations reflètent simplement les possibilités offertes par l'enseignement supérieur dans les grandes villes de la Fédération. Ces remarques ont en outre une portée limitée, compte tenu de la faiblesse des populations étudiées et des lacunes de l'information. Elles ne sauraient entamer le règne des bacharéis, au sens le plus large, sur les palais nationaux.

Il n'y a pas lieu de s'étonner que l'État brésilien, impérial ou républicain, ait été chercher ses cadres sur 1'“îlot lettré" émergé de l'océan d'analphabètes. Ainsi, les élites politiques ne recoupent pas totalement celles de la richesse. Les travées parlementaires sont peuplées de professions libérales, de fonctionnaires et d'intellectuels plus que de commerçants ou de planteurs. Ce phénomène traduit plutôt une division sociale du travail à l'intérieur des classes supérieures de la société brésilienne. Il introduit aussi un peu de souplesse dans une société fortement corsetée. Le bacharelismo permet à des hommes nouveaux de se hisser à un statut que leur naissance leur interdisait a priori.

\section{LA PROMOTION D'HOMMES NOUVEAUX}

Numa Pompílio de Castro, héros du roman de Lima BarretoNuma et la nymphe, est le type de ces petits-bourgeois qui, à force de privations, gagne ses galons universitaires et peut se lancer dans une carrière politique. Fils d'un petit 
fonctionnaire d'une province du nord, Numa a dû lutter pour devenirbacharel, puis, comme ce premier grade ne suffisait pas à suppléer l'obscurité de sa naissance et à lui mettre le pied à l'étrier, il a poussé ses études jusqu'au doctorat. Il peut ensuite mettre son habileté au service des caciques de la politique locale, devient le factoton indispensable de l'homme fort de l'obscur (et fictif) Etat de Sepotuba et épouse la fille de celui-ci avant d'atteindre la consécration d'un siège au Congrès fédéral. ${ }^{31}$

Le romancier décrit ainsi une voie classique d'ascension dans la société brésilienne. Le diplôme, allié à un beau mariage, permet aux Rastignactupiniquins de donner corps à leurs ambitions. Quelques trajectoires confirment la fiction de Lima Barreto. Quintino Bocaiúva (1836-1912), un des chefs des «républicains historiques», brille peu par ses origines sociales et doit interrompre prématurément son cursus à la faculté de São Paulo. Il devient cependant le "prince des journalistes" en mettant ses dons littéraires au service de la cause abolitionniste, puis de la République. Quintino Bocaiúva est l'auteur d'une vingtaine de drames et de mélodrames oubliés comme Onfália, Um padre louco ("un curé fou") ou Os mineiros da desgraça ("les mineiros du malheur"). Sa première épouse est la nièce du Vicomte de Macaé, sénateur de l'Empire, conseiller d'Etat et plusieurs fois ministre. ${ }^{32}$

Un compagnon de Quintino Bocaiúva, Nilo Peçanha (1867-1924) suit un parcours également exemplaire. Fils d'un commerçant d'origine portugaise de Campos, Nilo Peçanha est remarqué et protégé par le baron de Miracema. Nilo Peçanha fait son droit à Recife, adhère à l'idée républicaine, entre très activement en francmaçonnerie, et, surtout, dans une des plus nobles familles de planteursfluminenses. En 1895, Nilo Peçanha épouse en effet Anita de Castro Belisário de Souza, nièce du sénateur de l'Empire Francisco Belisário Soares de Souza, cousine germaine de Paulino Soares de Souza, Vicomte d'Uruguay. ${ }^{33}$ La suite de la carrière de Nilo Peçanha ne dément pas ces débuts prometteurs: il est successivement député fédéral, sénateur fédéral, plusieurs fois président de l'Etat de Rio de Janeiro, vice-

"Afonso Henriques Lima Barreto, Numa e a ninfa, Rio de Janeiro, Gamier, 1990 (lère éd., 1915).

32 Leonardo Leite Neto, éd., Catálogo biográfico dos senadores brasileiros, 1826-1989, Brasilia, 1986.

31 Leonardo Leite Neto, èd., Catälogo biográfico dos senadores brasileiros, 1826-1989, Brasilia, 1986. président et président de la République (1906-1910), ministre des affaires étrangères en 1917, et achève sa vie politique comme candidat malheureux à la présidence de la République en 1922.

Le troisième exemple est bahianais. En sortant de la Faculté de droit de Bahia, João Mangabeira (1880-1964), fils d'un pharmacien de Salvador, est parachuté dans la région d'Ilhéus où il travaille comme avocat. Il devient l'homme de confiance du Colonel Domingos Adami de Sá, gros planteur de cacao et chef politique de la région. João Mangabeira épouse une veuve dotée de nombreuses fazendas, et, grâce à son protecteur, il siège à l'assemblée législative de Bahia à partir de 1906 avant d'entrer à la Chambre fédérale en 1908. ${ }^{34}$ João Mangabeira sortira rapidement d'Ilhéus pour faire une carrière nationale.

João Mangabeira comme Nilo Peçanha, dont les gestions successives de l'État de Rio de Janeiro ont prouvé le pragmatisme et l'efficacité, sont des bacharéis, au sens où ils ont fait des études de droit, mais ils n'incarnent pas à proprement parler le bacharelismo.

\section{"RUI BARBOSA: UN HAUT-DE-FORME EN SENEGAMBIE" (OSWALD DE ANDRADE, MANIFESTE DE LA POESIE PAU BRASIL, 1924) ${ }^{35}$}

Lorsque les vertus de la Première République sont mises en doute, particulièrement dans les années 1920-, l'argumentaire qui avait servi à critiquer l'Empire est à peine actualisé. Alberto Torres, dont la pensée exerce une profonde influence sur la génération qui arrive au pouvoir après 1930 , voit dans le bacharelismo un handicap légué par la colonisation portugaise: "Savants, lettrés et juristes de Coimbra ont apporté chez nous de brillantes idées, des concepts théoriques, des formules juridiques, des institutions administratives,

" Gustavo Aryocara de Oliveira Falcon, Os coronéis do cacaut: raizes do mandonismo polifico em Illeéts 1890-1930, maitrise, Universidade Federal da Bahia, 1983, p. 93. Voir aussi l'article "Mangabeira, Jo 犭o", Dicionário Histórico-biográfico brasileiro, 1930-1983. Rio de Janeiro, Forense, vol. 3, pp. 2060-2065. 35 Oswald de Andrade, "Manifeste de la poésie Pau Brasil" Correio da Wanhä. São Paulo 18 mars 1924 cité Wodernidade Art brésilien du KY sècle Paris, Musée d'art moderne de la ville de Paris, 1987, p. 78. 
élaborés dans les centres européens. Avec un tel legs de doctrines et d'imitations, on a bâti un édicifice gouvernemental fait de matériaux étrangers, artificiel, bureaucratique. (...) les hommes publics -doctes parlementaires et administrateurs judicieux-n'étaient ni des hommes politiques, ni des hommes d'État; ils tissaient sur les réalités de notre vie une toile de discussions abstraites et théoriques; ils bataillaient sur des formules constitutionnelles françaises ou anglaises. ${ }^{936}$ Le constat d'Alberto Torres, qui ne perçoit guère de changement sous la République, n'a rien d'original, ni de très nouveau. Il corrobore des témoignages sur la vie parlementaire brésilienne du début de ce siècle.

Amilcar Viana Martins Filho écrit ainsi que les sessions de l'assemblée des députés du Minas Gerais ressemblaient "plus à un salon littéraire ou aux rencontres d'une société philanthropique qu'à une réunion de législateurs. ${ }^{137}$ On doit à José Vieira, journaliste spécialisé dans le compte rendu des séances du Congrès, quelques portraits de la représentation nationale en 1909: "Ceará: Francisco Souto, vieux latiniste et dévot. (...) Graco Cardoso: professeur de grec. (...) Pernambouc: Medeiros e Albuquerque: Fait des vers, de bons vers. Ecrit des contes et est journaliste politique. Alagoas: Eusébio de Andrade: membre d'une famille de poètes. ${ }^{n 38}$

Cette vision du personnel politique de la Première République ne doit cependant pas conduire à conclure au triomphe sans partage du bacharelismo. La carrière de Rui Barbosa, et le culte dont il a fait l'objet dans une partie de la jeunesse brésilienne de la première moitié du siècle, permettent d'apprécier la nature de l'esprit bacharel et les limites de son efficacité dans le système politique républicain.

36 "Scientistas, literatos e juristas da escola de Coimbra trouxeran, para o nosso meio, brillantes idéas, conceitos theoricos, fornulas juridicas, instituiçðes administrativas, estudados nos centros europeus. Com tal espolio de doutrinas e de imitaçoses, arçitectou-se um edificio governamental feito de materiae alleios, artificial burocratico () os homens publicos -doutos parlamentares e criteriosos administradoresa heios, a

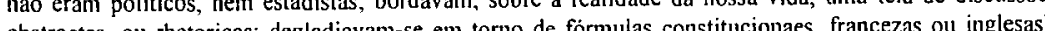
abstractas, ou rhetoricas: degladiavam-se em torno de formulas constitucionaes, francezas ou inglesas" Alberto Torres, A organizą̧ão nacional, São Paulo, Cia editora nacional, 1938 (lère éd., 1915), p. 57 17 Amilcar Viana Martins Filho, "Clientelismo e representação em Minas Gerais durante a Primeir República: uma critica a Paul Cammack", Dados, Rio de Janeiro, vol. 27. No. 2, 1984, p. 192.

18 José Vieira de Araíijo, $A$ Cadéia velha. Memória da Cámara dos deputados. Brasilia, Senado federal/Casa de Rui Barbosa, 1980 (lère éd., 1909), p.46.
Rui Barbosa (1849-1922), à la fois adulé et décrié, est l'incarnation reconnue du bacharelismo. comme en témoigne le fait que le poète moderniste Oswald de Andrade l'associe à "haut-de-forme en Sénégambie", l'expression d'une civilisation décalée.

Originaire de la ville de Bahia, Rui Barbosa fait ses études de droit à Olinda, puis à São Paulo, et s'installe en 1876 à Rio de Janeiro, où il fait fortune en tant qu'avocat. ${ }^{39}$ Fidèle à la tradition de sa famille, il s'engage assez tôt dans les combats du parti libéral, soutient les abolitionnistes. À la fin des années 1880 , il fait du fédéralisme un de ses chevaux de bataille et rejoint, pour cette raison, la conspiration républicaine de novembre 1889. Il occupe ainsi une position déterminante dans le gouvernement provisoire qui suit la Proclamation de la République et ne cantonne pas son action aux seules finances dont il a reçu la charge. C'est pourtant dans ce domaine que son passage aux affaires a laissé un souvenir cuisant. Convaincu de l'excellence pour le Brésil de l'exemple nordaméricain, grand admirateur d'Alexandre Hamilton, Rui Barbosa bouleverse le système financier brésilien de manière à stimuler l'activité économique et à favoriser le développement industriel. Il résulte de sa politique une croissance factice qui s'effondre au bout de quelques mois dans les faillites en séries et l'inflation. L'encilhamento -surnom de cette phase tumultueuse- entacha pour longtemps la confiance dans les capacités de Rui Barbosa. Après son départ du gouvernement en janvier 1891, Rui Barbosa continue à jouer un rôle important dans la politique nationale, depuis le Sénat où il siège longtemps, mais aussi lors de campagnes électorables mémorables. Il représente en outre le Brésil à la Conférence de La Haye en 1907 et en tire une grande publicité. "L'Aigle de la Haye" se fait le champion de la réforme des institutions républicaines et du respect des libertés publiques. Sa bibliographie complète, composée de discours, articles, commentaires et essais divers (y compris sur l'Affaire Dreyfus), occupe plusieurs pages de listing informatique. ${ }^{40}$ Afonso Arinos de Melo Franco le peint "enfermé dans sa formidable bibliothèque comme dans un fortin inexpugnable, d'où, à travers les meurtrières ouvertes entre lesétagères, dépêchait

39 Luis Viana Fillı, A vida de Rui Barbosa. Rio de Janeiro, Nova Fronteira, 1987.

to Leonardo Leite Neto, éd., Catálogo biográfico dos senadores brasileiros, 1826-1989, Brasilia. 1986, pp. 53-61. 
sur l'éventuel adversaire, abandonné en rase campagne, le feu et le fer de sa mitraille d'adjectifs compliqués et d'arguments ingénieux. ${ }^{21}$

Ses projets de révision constitutionnelle, Rui Barbosa les diffuse lors de ses candidatures désespérées à la présidence de la République en 1909 et en 1919. Les arrangements entre gouverneurs au pouvoir et les fraudes électorales généralisées ne laissent en effet aucune chance de l'emporter à un candidat "non officiel" comme Rui Barbosa. Les échecs et le panache de celui-ci ont sans doute contribué à forger la légende de ce don Quichote de la Belle Époque. Par son obstination à dénoncer les dévoiements de la République et grâce au fait qu'il n'ait pas exercé le pouvoir exécutif, Rui Barbosa demeure longtemps l'idole des étudiants en droit pour lesquels il représente "l'incarnation du droit lui-même». Son portrait, orné de la devise "avec la loi, par la loi, à l'intérieur de la loi", accueille longtemps les visiteurs dans le hall de la Faculté de Bahia. ${ }^{42}$

Archétype du bachárel et du bacharelismo, Rui Barbosa est en réalité marginalisé par la "politique des États" sur laquelle fonctionne la Première République depuis la présidence de Campos Sales (1898-1902). Le "plus illustre des Bahianais" est avare de visites dans sa terre natale. En avril 1919 , alors que Rui Barbosa en retrouve le chemin pour soutenir la candidature du juge Paulo Fontes au gouvernement de l'Etat, José Joaquim Seabra, chef de la faction adverse, lui adresse une lettre publique très mordante. Seabra inverse les rôles et attribue ironiquement à Rui Barbosa ses propres réalisations pour mieux faire ressortir l'inutilité du tribun bahianais: "Votre Excellence a oublié (...) de souligner qu'en foulant cette terre le 10 du mois, elle a trouvé pour l'accueillir une grande commission qui, émue, l'a remerciée d'avoir tantlutté

\footnotetext{
" "Rui e Pinheiro Machado, com seus Estados a varios dias de viagem de vapor, residian permanentemente em esplêndidas mansð̌es. O primeiro encerrado na sua formidável bibliotheca como num fortim inexpugnável, de onde, pelas seteiras abertas entre as estantes, despejava sobre o eventual adversário abandonado em campo raso o fogo e o ferro da sua inetralha de adjetivos complicados e argumentos engenhosos", Afonso Arinos de Melo Franco, Un estadista da República, Rio de Janeiro, José Olímpio/ USP, vol. 2, 1955, pp. 556-557.

42 Témoignage de Clemente Mariani déposé au secteur de "história oral" du CPDOC, transcription de la cassette No. 4 , p. 51 .
}

pour la construction des cuvres du port de cette capitale. (...) Peu après, en entrant dans la ville, une autre commission de riches et d'importants commerçants, touchée, a remercié Votre Excellence d'avoir fait détruire Santa Barbara, la ruelle de la Garapa, le grand urinoir qu'était tout le quartier commercial et d'avoir transformé tout ce tas d'ordures en rues aérées, larges et salubres. ${ }^{.23} \mathrm{Ce}$ sont là des travaux qui ont été accomplis sous l'administration de José Joaquim Seabra. L'apparition des gouverneurs sous la Première République fait surgir une nouvelle figure d'homme politique promis à un bel avenir au Brésil au bacharel qui parle et versifie commence à s'opposer le "governador que faz", l'homme politique qui agit.

La Première République constitue donc une période de transformation des élites politiques. Au moment où l'on stigmatise le formalisme et les sophismes des bacharéis, ceux-ci ont amorcé leur déclin au profit de nouvelles figures d'administrateurs et d'hommes politiques.

De la Révolution de 1930 à 1945, -avec une brève exception entre 1933 et 1937-, le Congrès fédéral, haut lieu du bacharelismo, est supprimé des institutions brésiliennes. La notion de concours est introduite pour les recrutements administratifs. Des équipes de technocrates spécialisés sont censés remplacer les mandarins individualistes, écartés des affaires. Le bacharelismo semble enterré par le populisme des années 1950. Ainsi triomphe durablement l'homme politique "qui fait" et passe ses mandats à inaugurer des réalisations de toute sorte et à dévoiler des plaques qui commémorent ses bienfaits. L'élection et la réélection de Fernando Henrique Cardoso à la présidence de la République en 1994 et 1998 suggèrent cependant la résurgence du bacharelismo, à moins qu'il ne s'agisse de l'apparition d'un néo-bacharelismo, dans lequel Tocqueville et Weber ont remplacé Plutarque et Lamartine pour rendre compte des oscillations de l'opinion publique et des effets de la mondialisation sur le panier de la ménagère.

43 Lettre du 15 avril 1919, Francisco Borges de Barros, Dr. José Joaquim Seabra: sua vida, sua obra na Repiblica, Bahia, Imprensa nacional, 1931, p. 494. 